\title{
Cavitary Pneumonia and Skin Lesions
}

\author{
Moncef Belhassen-Garcia MD, Virginia Velasco-Tirado MD, Lucia Alvela-Suárez MD, \\ Maria del Carmen Fraile-Alonso MD, Adela Carpio-Pérez MD PhD, \\ and Javier Pardo-Lledias MD PhD
}

\begin{abstract}
Tularemia is a worldwide zoonosis caused by Francisella tularensis. The most frequent forms of tularemia are ulceroglandular, followed by typhoidal forms, glandular, and oculoglandular. Respiratory involvement is an uncommon presentation. Cutaneous lesions secondary to respiratory infections occur in $30 \%$ of cases. We present a case of tularemia with cavitary pneumonia and skin lesions. Key words: tularemia; cavitary pneumonia; skin lesions. [Respir Care 2012;57(3):457-459. (C) 2012 Daedalus Enterprises]
\end{abstract}

\section{Introduction}

Tularemia is a worldwide zoonosis caused by Francisella tularensis, with 2 serotypes, presenting different morbidity-mortality and geographic distribution. Arthropods (ticks and deer flies) are the main transmission vector, and small animals (rabbits, hares, and muskrats) serve as reservoir hosts. ${ }^{1} F$. tularensis is probably one of the most infectious organisms known. Cavitary pneumonia is an uncommon manifestation on its clinical spectrum, frequently acquired via inhalation. Cutaneous lesions secondary to respiratory infections occur in less than one third of the cases, attributed to immune modulation. ${ }^{2}$ Erythema nodosum and erythema multiforme are described as typical skin alterations. As these lesions appear in the second phase of the illness and they are similar to those in sec-

Drs Belhassen-Garcia, Velasco-Tirado, Alvela-Suárez, Carpio-Pérez, and Pardo-Lledias are affiliated with Servicio Medicina Interna, Unidad de Enfermedades Infecciosas, Hospital Universitario de Salamanca, Salamanca, Spain. Drs Belhassen-Garcia, Velasco-Tirado, and Pardo-Lledias are affiliated with the Centro de Investigación de Enfermedades Tropicales, Departamento de Biologia Animal y Parasitologia, Facultad de Farmacia, Universidad de Salamanca, Salamanca. Spain. Dr Fraile-Alonso is affiliated with Servicio de Dermatologia, Hospital Universitario de Salamanca, Universitario de Salamanca, Salamanca, Spain.

The authors have disclosed no conflicts of interest.

Correspondence: Lucia Alvela-Suárez MD, Servicio Medicina Interna, Hospital Universitario de Salamanca, Paseo San Vicente s/n 37007 , Salamanca, Spain. E-mail: luciaalvela@hotmail.com.

DOI: $10.4187 /$ respcare. 01188 ondary syphilis, they have been called tularemids. ${ }^{3} \mathrm{We}$ present a case of tularemia with skin lesions secondary to pneumonia.

\section{Case Report}

A 69-year-old woman was admitted with abdominal pain in the right upper quadrant and temperature of $39^{\circ} \mathrm{C}$ of one week's evolution. She did not present any relevant medical history. She had not traveled abroad, and she had daily contact with wild rabbits on her farm. She complained only of mild cough. She did not feel chest pain, hemoptysis, or shortness of breath.

At first the patient had developed fever without cutaneous lesions or lymphadenopathy. The auscultation revealed crackles in the base of the right lung. She also showed Murphy's sign. Laboratory studies showed a white-bloodcell count of $6,950 / \mathrm{mL}$ and a neutrophil level of $5,320 / \mathrm{mL}$. The platelet count was normal, the level of C-reactive protein was above $9 \mathrm{mg} / \mathrm{dL}$, and the erythrocyte sedimentation rate was $110 \mathrm{~mm} / \mathrm{h}$. The alanine aminotransaminase and the aspartate aminotransferase levels were $41 \mathrm{U} / \mathrm{L}$ and $33 \mathrm{U} / \mathrm{L}$, respectively. The chest $\mathrm{x}$-ray did not show pulmonary infiltration. The abdominal ultrasonography did not detect any biliary or hepatic problems.

We started an empiric treatment with amoxicillin-clavulanic $2 \mathrm{~g}, 3$ times a day. The blood, sputum, and urine culture with a standard aerobic and anaerobic medium were negative for pathogens. Intradermal tuberculin skin test was $<5 \mathrm{~mm}$. The Legionella and pneumococcal urinary antigens were negative. Also, Chlamydia species, $M y$ coplasma species, and F. tularensis enzyme immunoas- 


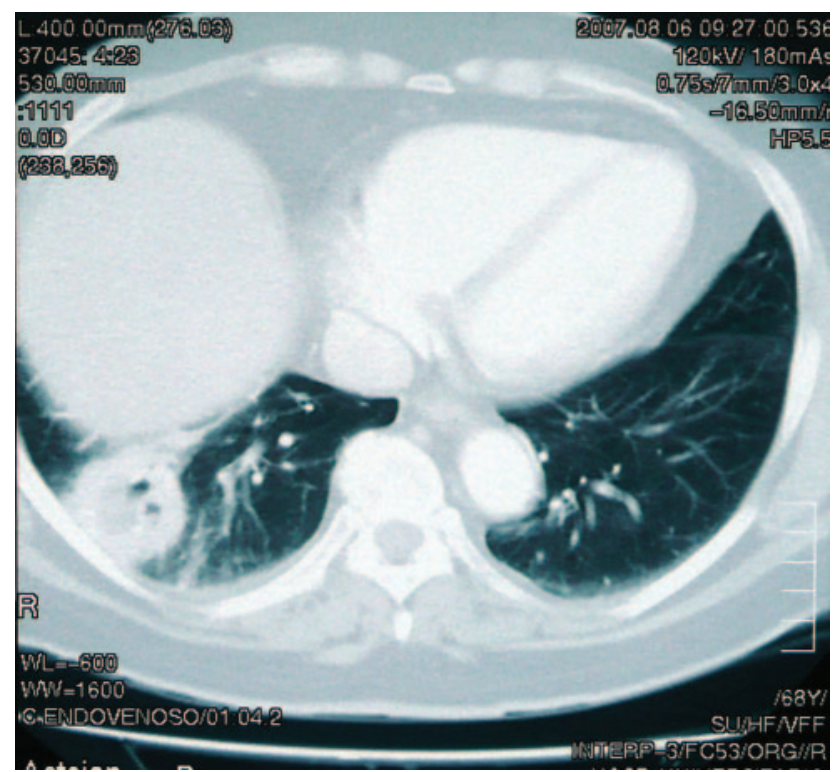

Fig. 1. Thoracic computed tomography. There is a $4 \mathrm{~cm}$ cavitary lesion in the lower right lobe.

says were negative. Ricketssia species and Coxiella species indirect immunofluorescence tests were negative at first. Serum agglutination test of Brucella species and fluorescent treponemal antibody-absorption of Treponema species were also negative. Moreover, we found seronegativity in the test for human inmunodeficiency virus, cytomegalovirus, Epstein-Barr virus, hepatitis B virus, hepatitis $\mathrm{C}$ virus, and hepatitis A virus. We carried out a study of tumor markers and autoantibodies for autoimmune disease, and all the results were negative.

The patient continued presenting high fever daily, and a thoracic computed tomography was performed (Fig. 1), showing a $4 \mathrm{~cm}$ cavitary lesion in the lower right lobe. At that moment the patient developed a $1-3 \mathrm{~cm}$ purple papular eruption with pain and pruritus on the palms of the hands (Fig. 2). Thus, on day 4, a second antimicrobial, ciprofloxacin $500 \mathrm{mg}$ was initiated. A biopsy of skin lesion showed an unspecific interstitial and perivascular inflammation. Skin culture was also negative. Three days after starting ciprofloxacin, the patient presented a remission of the fever, and the skin lesions showed a peripheral desquamation (Fig. 3). Four weeks later we detected an almost complete remission of the cavitary pneumonia. Then we carried out a second enzyme immunoassay test for tularemia, with a titer of $1 / 2,560$.

\section{Discussion}

Tularemia is a zoonotic infection caused by $F$. tularensis, which is a Gram-negative, pleomorphic rod. It was first identified as a cause of human disease in 1914. It has

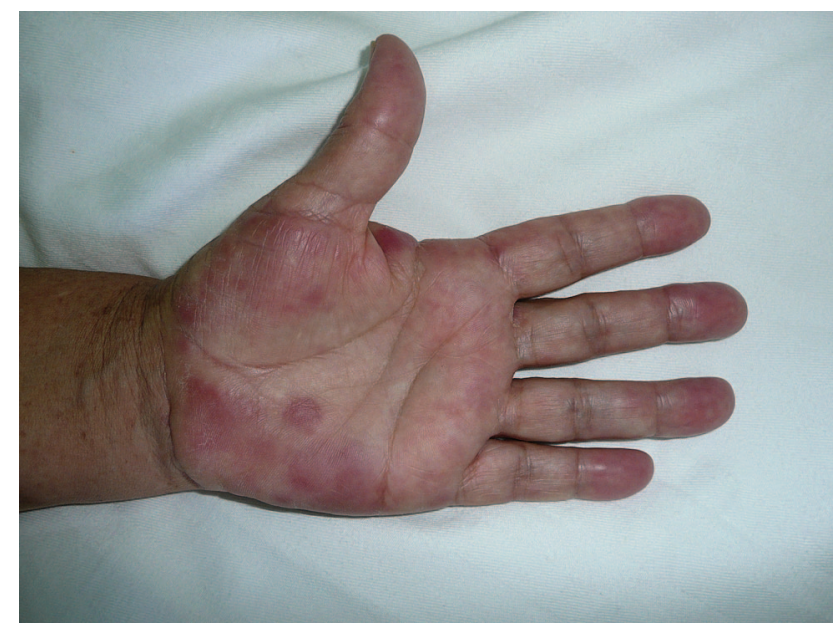

Fig. 2. Skin lesions. Purple papular eruption with pain and pruritus on the palms of the hands.

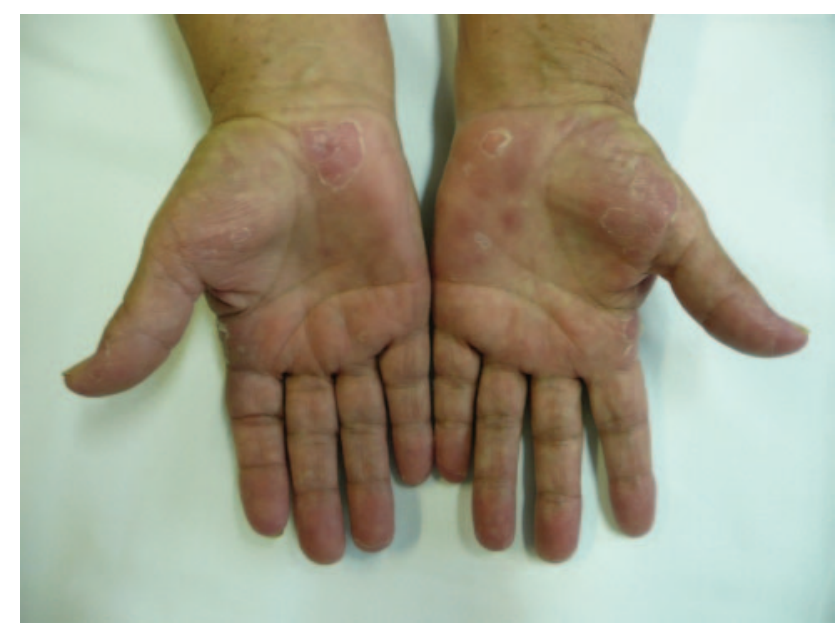

Fig. 3. Evolution of skin lesions. Skin lesions with peripheral desquamation.

been designated a Category A bioterrorism agent by the Centers for Disease Control, because of its unique ability to disseminate and its potential use as an agent of bioterrorism. ${ }^{4}$ The diagnosis of tularemia is usually made in an epidemiologically and clinically compatible setting, and the seroconversion of $F$. tularensis occurs between 4 and 6 weeks later. Enzyme immunoassay for tularemia has a sensitivity of $95.7 \%$ and specificity of $96 \%$. Although there are differences between studies, the most frequent forms of tularemia are ulceroglandular (61.3\% of cases), followed by typhoidal forms (20.4\% of cases), glandular (9.2\% of cases), and, finally, oculoglandular (4.2\% of cases). ${ }^{5}$ The incubation period varies from a few hours to 11 days, with an average time of 3 days. ${ }^{6}$

Primary pneumonia is an infrequent disease $(3.5 \%),{ }^{5}$ although it may appear in 10-15\% of ulceroglandular cases and in $30-80 \%$ of typhoidal cases, due to a hematogenous 
dissemination. ${ }^{7}$ There are no specific symptoms, signs, or $\mathrm{x}$-ray patterns for pulmonary tularemia. Cavitary pneumonia, such as our patient presented, is not an exceptional form. After a pulmonary biopsy, Maranan et al found areas of caseous necrosis in a 14-year-old boy with a necrotizing pneumonic tularemia, similar to those caused by Mycobacterium tuberculosis. ${ }^{7,8}$

The primary skin alterations are caused by $F$. tularensis getting through small lesions in the skin. The classic presentation is a cutaneous ulcer, frequently associated with regional lymphadenopathies. Furthermore, $8-35 \%$ of patients present secondary skin lesions. The average delay between the onset of the first symptoms and the appearance of the secondary skin lesions is 11 days (range 3 days to several weeks). The most common eruptions are papular or vesiculo-papular (42\%), erythema nodosum $(22 \%)$, erythema multiforme (6\%), acne-like eruptions $(6 \%)$, and urticaria (2\%). ${ }^{9}$ Distinguishing between some infections with skin lesions and tularemia can be difficult by visual inspection alone. ${ }^{4}$

In our case, as we showed above, the secondary skin eruption began 11 days after the onset of the symptoms. The eruption was papular and the distribution was exclusively found in the palms of the hands. In this sense, there are some authors who would classify these lesions as tularemids, due to the similarities with a lesion secondary to syphilis. ${ }^{3}$ The cutaneous biopsy of these lesions in our patient was unspecific, as usual. It showed an infiltrated perivascular and interstitial inflammation. The lesions in our patient disappeared within 4 weeks, after a successful treatment with ciprofloxacin.

$F$. tularensis is rarely seen on Gram-stained smears or in tissue biopsies, and does not grow in routinely plated cultures. It may be recovered using supportive media. Thus, if an attempt is made to culture $F$. tularensis from clinical specimens, the laboratory should be notified so that personnel can optimize growth conditions and take proper precautions to reduce the risk to laboratory staff. ${ }^{10}$

No prospective controlled clinical trials have defined the efficacy of different drug treatments or the optimal duration of therapy for tularemia. Nevertheless, most authors believe that streptomycin is the drug of choice. ${ }^{11}$

In summary, it is essential to recognize the different manifestations of the disease by the physicians in endemic or epidemic areas to avoid misdiagnosis and the prescription of inadequate antibiotics. Because of the threat of biological terrorism, medical staff must be aware of the possibility of tularemia presenting with skin lesions and fever. ${ }^{4}$

\section{REFERENCES}

1. Tarnvik A, Berglund L. Tularaemia. Eur Respir J 2003;21(2):361373.

2. Evans ME, Gregory DW, Schaffner W, McGee ZA. Tularemia: a 30-year experience with 88 cases. Medicine (Baltimore) 1985;64(4): 251-269.

3. Galle R, Chervonaz B, Texier J. Cutaneous nodular type tularemia. Nodular tularemides. Ann Dermatol Venereol 1982;109(9):767-768.

4. Byington CL, Bender JM, Ampofo K, Pavia AT, Korgenski K, Daly $\mathrm{J}$, et al. Tularemia with vesicular skin lesions may be mistaken for infection with herpes viruses. Clin Infect Dis 2008;47(1):e4-e6.

5. Perez-Castrillon JL, Bachiller-Luque P, Martin-Luquero M, MenaMartin FJ, Herreros V. Tularemia epidemic in northwestern Spain: clinical description and therapeutic response. Clin Infect Dis 2001; 33(4):573-576.

6. Eliasson H, Back E. Tularaemia in an emergent area in Sweden: an analysis of 234 cases in five years. Scand J Infect Dis 2007;39(10): 880-889.

7. Maranan MC, Schiff D, Johnson DC, Abrahams C, Wylam M, Gerber SI. Pneumonic tularemia in a patient with chronic granulomatous disease. Clin Infect Dis 1997;25(3):630-633.

8. Kozak AJ, Hall WH, Gerding DN. Cavitary pneumonia associated with tularemia. Chest 1978;73(3):426-427.

9. Syrjala H, Karvonen J, Salminen A. Skin manifestations of tularemia: a study of 88 cases in northern Finland during 16 years (19671983). Acta Derm Venereol 1984;64(6):513-516.

10. Overholt EL, Tigertt WD, Kadull PJ, Ward MK, Charkes ND, Rene $\mathrm{RM}$, et al. An analysis of forty-two cases of laboratory-acquired tularemia. Treatment with broad spectrum antibiotics. Am J Med 1961;30:785-806.

11. Enderlin G, Morales L, Jacobs RF, Cross JT. Streptomycin and alternative agents for the treatment of tularemia: review of the literature. Clin Infect Dis 1994;19(1):42-47. 